Chirurgia (2018) 113: 289-290

No. 3, May - June

Copyright $\odot$ Celsius

http://dx.doi.org/10.21614/chirurgia.113.3.289

\title{
The Future of Pancreatic Surgery
}

\section{Sorin T. Barbu}

$4^{\text {th }}$ Surgery Department, University of Medicine and Pharmacy "Iuliu Hatieganu” Cluj-Napoca, Romania

\author{
Corresponding author: \\ Sorin T. Barbu, MD, PhD \\ Chair of the 4th Surgery Department \\ University of Medicine and Pharmacy \\ "Iuliu Hatieganu" Cluj \\ President of the Romanian Pancreatic \\ Club \\ 18, Republicii Street,, Cluj-Napoca \\ 400015, Romania \\ E-mail: tbarbu@umfcluj.ro \\ barbu@pancreasclub.ro
}

Pancreatic surgery has undergone a relevant progress during the last two decades. Specialized centers with multidisciplinary expertise have led to improved patients care with decreased morbidity and mortality. In acute pancreatitis, paradigms have shifted towards conservative and minimally invasive management. In chronic pancreatitis, parenchymasparing resections have been widely accepted. Cystic pancreatic lesions - especially IPMN - have produced a great interest in surgical practice, and several guidelines have already been published, but the debate continues. In pancreatic cancer treatment, surgical resection techniques have improved, the impact of adjuvant treatment has been demonstrated in large multicenter trials, neoadjuvant approaches seems extremely promising, and evaluation trials continue. The application of minimal-invasive surgery (laparoscopic, robotic) to pancreatic resections had occurs slowly, but steadily. Regarding evidence-based medicine, consensus definitions on main complications of pancreatic resections, standards for resections and their reporting have been published by the International Study Group for Pancreatic Surgery. According to recent randomized trials and advances in medical treatment, several surgical dogmas have been refuted. The number of ongoing high-quality trials on unsolved questions in pancreatic surgery is continuously increasing.

In Acute Pancreatitis (AP), surgical management has decreased, and open necrosectomies tend to become rare procedures in surgical departments. The PANTER randomized controlled trial (RCT) - 2010 -has taken down open necrosectomy from its "gold standard position, replacing it with the step-up approach (percutaneous drainage followed by VARD). The TENSION trial (2017) proved endoscopic step-up approach not to be superior to the surgical step-up approach, but even if statistically not significant, the endoscopic treatment seems to be less expensive. This trend is likely to even increase in the future. The outcomes of interventional and minimally invasive procedures are more favorable and less aggressive. Regarding the Future, surgeons of 
the next generation will probably consider acute pancreatitis as a generally non-surgical disease, with extremely rare indications foropen surgery.

Surgical procedures in Chronic Pancreatitis (CP) have become safe and efficient due to the standardization of parenchyma-sparing procedures, the experience of surgeons in specialized centers, and optimization of perioperative treatment. For most patients with the typical finding of an inflammatory pseudo-tumor in the pancreatic head, the method of choice is a duodenum-preserving pancreatic head resection. Even if the randomized controlled trials have proven the long-term advantages of a surgical approach compares to endoscopic interventions, we had a dramatically decrease in the number of CP patients addressed to surgery (from 70\% twenty years ago, to $11 \%$ today). The evidence-based superiority of surgery should be respected in the interdisciplinary treatment of these patients. Looking at the Future, the role of surgery in the management of CP patients will increase if clinical evidence is respected, or, it will be reserved for advanced CP patients when all endoscopic procedures have failed.

Cystic Lesions of the pancreas are probably the most dynamic field of pancreatic surgery in the future. Especially the treatment of intraductal papillary mucinous neoplasias (IPMN) has been and is currently under controversial debate. For the Future, a longtime follow-up and several RCTs are needed to have more accurate data on when to resect and when to follow-up.

Regarding Pancreatic Cancer, every physician knows the dictum "surgery is the only chance for cure", which was changed into "surgery is the first step to cure" when the RCTs have demonstrate the benefit of adjuvant therapy. When we'll have significant RCTs on neoadjuvant therapy, the dictum will change again. Pancreatic surgery has been well-standardized and has become safe routine in many centers. This implies, that also extended approaches as vascular and multivisceral resections are performed increasingly. Regarding resection techniques in pancreatoduodenectomy, the "artery-first" and "mesopancreas" excision, have been described and established. When reconstruction is considered, handling of the pancreatic remnant is still not standardized on a high level of evidence. Several anastomoses are currently used, including different types of pancreatico-jejunostomy and pancreatico-gastrostomy. No high-level studies have yet shown a superiority of any technique. Although the preservation of the pylorus has been regarded as a standard in pancreato-duodenectomy, this is associated with a high rate of gastric emptying delay. Therefore, an increasing number of studies had deal with this problem and the concept of pylorus ring resection has been introduced and may offer a benefit. Despite great efforts to improve results of distal pancreatectomy in terms of post-operative pancreatic fistula, this problem is unsolved to date and probably one of the major challenges in the future. The DISPACT trial as a multicenter study has shown that remnant closure of the pancreas by suture or stapler leads to equal rates of leakage, which suggests that merely technical modification of this operative step may not be further effective. In the Future of pancreatic cancer surgery, we need an early detection (efficient screening), which will allow a 5 years survival of over $50 \%$; we need molecular and genetic biomarkers that will stratify the patients according to prognosis and responsiveness to chemotherapy; and finally, we need new efficient oncologic medications.

Regarding Laparoscopic pancreatic surgery, recent studies have shown that distal pancreatectomy can be performed with good results, and it represents already the standard for benign or borderline lesion of the body and tail. However, randomized studies are still lacking and selection bias is a major problem in the discussion of laparoscopic versus open proceedings, especially in pancreatic cancer patients. Laparoscopic and Robotic pancreatoduodenectomy - although increasingly performed by some centers - seems to be effective procedures in carefully selected patients. The Future of laparoscopic and robotic pancreatic surgery is bright, but time and money will be needed so that they become standard procedures in pancreatic surgery.

As surgeons, we need in the Future to further decrease mortality and morbidity of pancreatic resections. Preoperative risk stratification allows rational selection of patients amenable for surgical therapy and prediction of postoperative morbidity. The Postoperative Morbidity Index is a novel tool that allows utility based quantification of postoperative morbidity at the cohort level. And what we really need, it is Centralization, not only for surgery, but a multidisciplinary centrali-zation in specialized centers that will offer best care to the patients with pancreatic diseases. 